an immune-mediated hemolytic reaction. In our case, it was not clear why the DAT was negative. Perhaps almost all of the sensitized RBCs were rapidly cleared from the patient's circulation during the hemolytic episode and decreased to undetectable levels.

To our knowledge, this is the third reported case of DHTR resulting from anti-Jka after cardiac surgery, but it is the first case in which the development of this antibody occurred during pregnancy.

During the past 2 decades, a trend toward a decrease in the incidence of DHTRs occurred according to the Mayo Clinic's data. $^{2}$ A number of factors could influence the incidence of DHTRs, including changes in the average length of stay for inpatients, the average number of RBC transfusions per inpatient, and the adoption of new antibody detection systems. ${ }^{2,3}$ Cumulative data from 8 years (1996-2004) of Serious Hazards of Transfusion (SHOT) show that the incidence of DHTRs remains stable, accounting for $10.2 \%$ of all transfusion-related reactions. Responsible antibodies were consistent with those reported in the literature, with Kidd remaining the major cause of DHTRs (42\%). ${ }^{4}$
Development of new screening techniques to improve the detection of extremely low levels of Kidd antibodies is mandatory, especially for patients undergoing cardiac surgery, where the transfusion requirements are increased. Consideration should be given to issuing antibody cards or similar information to all patients with clinically significant red cell antibodies.

\section{References}

1. Blood groups: In: Brecher ME, editor. Technical manual, 14th ed. Bethesda: American Association of Blood Banks; 2002. p. 327.

2. Pineda AA, Vamvakas CE, Gorden DL, Winters LJ, Moore BS. Trends in the incidence of delayed hemolytic and delayed serologic transfusion reactions. Transfusion. 1999;39:1097-103.

3. Schonewille H, van de Watering LGM, Loomans ED, Brand A. Red blood cell alloantibodies after transfusion: factors influencing incidence and specificity. Transfusion. 2006;46:250-6.

4. Serious Hazards of Transfusion annual report 2004. Manchester: Serious Hazards of Transfusion Office; 2005.

\title{
Sternal tuberculosis after sternotomy for coronary artery bypass surgery: A case report and review of the literature
}

\author{
Kirun Gopal, MS, ${ }^{\text {a,c }}$ Austin Raj, DNB, ${ }^{a}$ M. R. Rajesh, MBBS, ${ }^{a}$ S. K. Prabhu, FRCS, \\ and Jayasree Geothe, MD, ${ }^{\text {b } K e r a l a, ~ I n d i a ~}$
}

\begin{abstract}
Clinical Summary
A 72-year-old man presented with upper back pain for 1 month and swelling over the sternum of 4 days' duration. He had no constitutional symptoms. One year earlier he had undergone coronary artery bypass surgery with an uneventful postoperative recovery. Clinical examination revealed a fluctuant swelling over the body of the sternum. There was mild tenderness but no erythema. Radiography of the chest and thoracic spine showed normal results (Figure 1). He had a total white blood cell count of $10,000 / \mathrm{mm},{ }^{3}$ with a differential count of $68 \%$ neutrophils and $30 \%$ lymphocytes, and an erythrocyte sedimentation rate of $105 \mathrm{~mm} /$ hour. Drainage of sternal abscess and removal of underlying possibly infected sternal wires were performed. Intraoperatively, a
\end{abstract}

From the Departments of Cardiovascular and Thoracic Surgery and Pathology, ${ }^{\text {b }}$ SUT Hospital, Trivandrum, Kerala, India.

Received for publication Dec 14, 2006; accepted for publication Jan 8, 2007.

Address for reprints: Kirun Gopal, Add: Chathayam, Ancheril Gardens, Gandhinagar PO, Kottayam 686008, Kerala, India (E-mail: kirungopal@ yahoo.com).

J Thorac Cardiovasc Surg 2007;133:1365-6

$0022-5223 / \$ 32.00$

Copyright $\odot 2007$ by The American Association for Thoracic Surgery

doi:10.1016/j.jtcvs.2007.01.022 large abscess cavity $(3.5 \times 3 \mathrm{~cm})$ was detected in the body of the sternum unrelated to the sternal wires. The cavity was lined with infected granulation tissue extending to the inner table of the

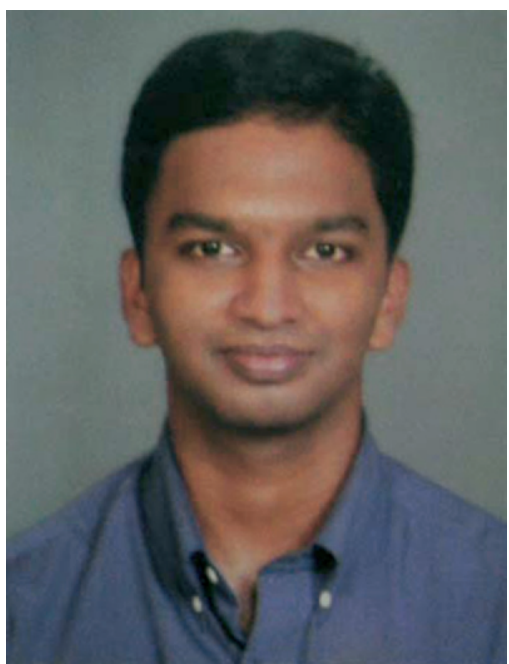

Dr Gopal sternum. The granulation tissue was curetted until healthy bleeding bony surface was observed. The pectoralis major muscle was mobilized to cover the defect, and the wound was closed in layers over a suction drain. The patient had an uneventful postoperative recovery and was relieved of back pain. The curetted tissue was sent for histopathology and bacterial culture. Histology revealed a caseating granulomatous lesion with sequestrum formation consistent with tuberculous osteomyelitis (Figure 2); the routine culture was negative, whereas BACTEC culture revealed mycobacterium tuberculosis (TB). On a 4-drug antituberculous regimen, the patient is doing well at 6 months.

\section{Discussion}

TB of the sternum has rarely been reported in the literature. Primary sternal TB is relatively more common compared with sternal TB secondary to surgery. A Medline search for cases of 


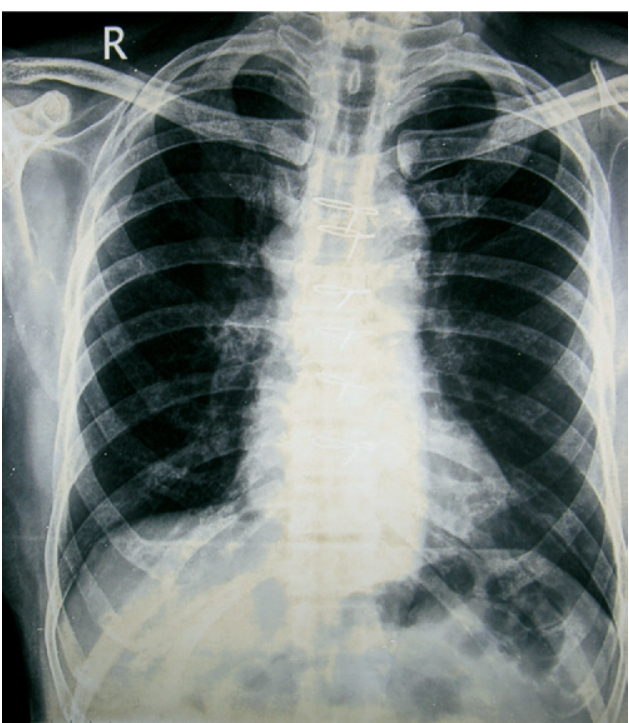

Figure 1. Chest x-ray showing no obvious bony abnormalities.

sternal TB detected after sternotomy for heart surgery revealed only 4 case reports, highlighting the rarity of the condition. ${ }^{1-4}$

$\mathrm{TB}$ of the sternum is rare (1.5\% of osteoarticular TB). ${ }^{5} \mathrm{Up}$ to one third of such patients had a detectable tuberculous lesion in other parts of the skeleton or in the lungs. Our patient had no evidence of skeletal $\mathrm{TB}$, and his chest radiography showed no evidence of prior TB. Sternal TB often presents as an abscess or sinus in the region of the sternum. Radiographic findings of sternal TB occur much later than those taken with clinical measurements. Radiography may reveal irregular cavities and areas of destruction in the bone with little surrounding sclerosis (honeycomb appearance), and there may be soft tissue swelling. The cavities may contain soft feathery sequestra, and the bone may show subperiosteal new bone formation. This clinicoradiologic picture needs to be differentiated from chronic pyogenic osteomyelitis, Brodie's abscess, tumors, or rare granulomatous conditions. Diagnosis is usually made by culture of the discharge or by histopathologic examination or culture of the resected tissue. Initial culture reports may be negative, and in cases with recurrent nonhealing wounds, repeat cultures for TB should be advised. Tuberculous osteomyelitis responds well to antitubercular drugs. Lesions can be seen radiologically to start healing by 6 months. Response depends on the stage of disease when treatment was started.

Surgical excision is justified in refractory cases, whenever there is a doubt in diagnosis, in the presence of a large abscess in the soft tissues around the involved bone, or to remove large sequestrum.

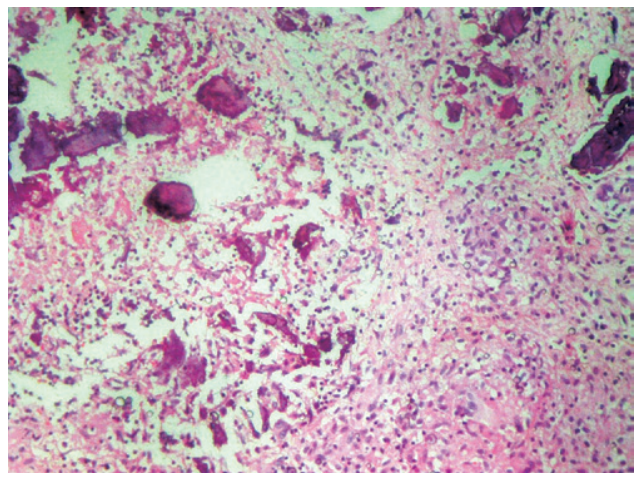

Figure 2. Multiple caseating granulomatous lesions and necrotic bone.

Surgical treatment for tuberculous osteomyelitis of the sternum involves extensive debridement and curettage of all involved tissue. The defect can be closed primarily if possible or more commonly by mobilization of the overlying pectoralis major as a flap. Other methods described in the literature include vacuum-assisted closure with a drain or use of a pedicled omental flap to help close the defect.

This case is being reported to make clinicians aware of this rare cause of sternal wound infection after sternotomy. Because of the rarity of the condition and often mild local symptoms, there may be a delay in diagnosis. The possibility of sternal TB should be kept in mind in any patient with a nonhealing ulcer or abscess in the sternal region. With awareness of the condition, diagnosis can be made preoperatively and antituberculous treatment instituted. Surgery with a complete course of antituberculous treatment gives good long-term results.

\section{References}

1. Rubinstien EM, Lehmann T. Sternal osteomyelitis due to Mycobacterium tuberculosis following coronary artery bypass surgery. Clin Infect Dis. 1996;23:202-3

2. Rivas P, Gorgolas M, Gimena B, Sousa J, Fernandez-Guerrero ML. Sternal tuberculosis after open heart surgery. Scand J Infect Dis. 2005; 37:373-4.

3. Sipsas NV, Panayiotakopoulos GD, Zormpala A, Thanos L, Artinopoulos C, Kordossis T. Sternal tuberculosis after coronary artery bypass graft surgery. Scand J Infect Dis. 2001;33:387-8.

4. Aggarwal B, Kamath S, Shatapathy P. Tubercular sternal osteomyelitis and mediastinitis after open heart surgery. Indian Heart J. 1997;49: 313-4.

5. Tuli SM. Tuberculosis of rare sites, girdle and flat bones. In: Tuli SM, editor. Tuberculosis of the Skeletal System (Bones, Joints, Spine and Bursal Sheaths), 2nd ed. Delhi: Jaypee Brothers Medical Publishers (P) Ltd; 2000:159-60. 